\title{
Can measurements of 2HDM parameters provide hints for high scale supersymmetry?
}

\author{
Ipsita Saha*† \\ Kavli IPMU (WPI), University of Tokyo, Kashiwa, 277-8583, Japan \\ E-mail: ipsita.saha@ipmu.jp
}

\begin{abstract}
The absence of any sign of supersymmetric (SUSY) particle at the current run of the LHC has pushed the SUSY breaking scale much above the electroweak (EW) scale. We show that considering the two-Higgs-doublet models (2HDM) as the effective low energy manifestation of such high scale SUSY, one can still get a hint for the SUSY breaking scale from the measurement of the $2 \mathrm{HDM}$ parameters at the low energy.
\end{abstract}

Corfu Summer Institute 2019 "School and Workshops on Elementary Particle Physics and Gravity" (CORFU2019)

31 August - 25 September 2019

Corfù, Greece

\footnotetext{
${ }^{*}$ Speaker.

${ }^{\dagger}$ Work done in collaboration with G. Bhattacharyya, D. Das, M. Jay Pérez, A. Santamaria and O. Vives.
} 


\section{Introduction}

In the post-Higgs discovery era, the Large Hadron Collider (LHC) has not seen any sign of new physics yet. Moreover, the LHC Higgs data has shown an affinity towards the Standard Model (SM) values[1,2]. This has already pushed the scale of many Beyond the Standard Model (BSM) scenarios to higher values if not discarded at all. Therefore, BSMs that are able to attain an alignment limit $[3,4,5,6,7,8]$, where a SM-like Higgs can be recovered, hold the key for future survival. The two-Higgs-doublet models (2HDM)[9, 10] extensions are among those. Besides providing an alignment limit, $2 \mathrm{HDMs}$ also have the desirable property that the oblique electroweak $\rho$ parameter remains unity at the tree level. Interestingly, the scalar sector of the minimal supersymmetric models (MSSM) [11, 12, 13, 14, 15, 16] are structured around two-Higgs doublets.

In this proceeding [17], we consider that the $2 \mathrm{HDM}$ is an effective low-energy manifestation of some fundmental ultraviolet (UV) complete scenario with an enhanced symmetry at the high scale. Therefore, the interesting question would be to know if the measurement of 2HDM parameters at the low-energy scale can provide us any hint of its embedding UV scenario that has an inaccssible mass scale. We show that by studying the renormalization group (RG) running of the 2HDM parameters, we would be able to test. In particular, we consider the MSSM framework[18, 19, $20,21,22,23,24,25,26]$ as the UV completer scenario with considerable high SUSY breaking scale. We follow a bottom-up approach and assume the 2HDM scalar masses and mixings at the low energy scale beforehand. Then we run the Higgs quartic couplings using the 2HDM RG equations $[27,28,29]$ and check if they satisfy the SUSY boundary conditions at some higher scale. Our approach is preferable as it is independent of the details of the underlying theory which are hidden in the matching conditions at the high energy scale.

\section{Effective two-Higgs-doublet model}

The two-Higgs-doublet model is built upon two $\mathrm{SU}(2)$ doublet scalars (with hypercharge $Y=$ $+1), \phi_{1}$ and $\phi_{2}$, and the most general gauge-invariant scalar potential can can be written as [3]

$$
\begin{aligned}
V & =m_{11}^{2} \phi_{1}^{\dagger} \phi_{1}+m_{22}^{2} \phi_{2}^{\dagger} \phi_{2}-\left(m_{12}^{2} \phi_{1}^{\dagger} \phi_{2}+\text { h.c. }\right) \\
& +\frac{\lambda_{1}}{2}\left(\phi_{1}^{\dagger} \phi_{1}\right)^{2}+\frac{\lambda_{2}}{2}\left(\phi_{2}^{\dagger} \phi_{2}\right)^{2}+\lambda_{3}\left(\phi_{1}^{\dagger} \phi_{1}\right)\left(\phi_{2}^{\dagger} \phi_{2}\right)+\lambda_{4}\left(\phi_{1}^{\dagger} \phi_{2}\right)\left(\phi_{2}^{\dagger} \phi_{1}\right) \\
& +\left[\frac{\lambda_{5}}{2}\left(\phi_{1}^{\dagger} \phi_{2}\right)^{2}+\left(\lambda_{6}\left(\phi_{1}^{\dagger} \phi_{1}\right)+\lambda_{7}\left(\phi_{2}^{\dagger} \phi_{2}\right)\right)\left(\phi_{1}^{\dagger} \phi_{2}\right)+\text { h.c. }\right] .
\end{aligned}
$$

These three bilinear mass parameters and 7 quartic couplings can be understood as the $\overline{\mathrm{MS}}$ parameters that can arise at the EW scale from a more complete theory at higher energies. It is evident that the potential has an additional U(1) global symmetry [30], If the parameters $m_{12}, \lambda_{5}, \lambda_{6}, \lambda_{7}$ are all zero while the $\mathrm{U}(1)$ is broken and there remains only an unbroken discrete $Z_{2}$ symmetry If $m_{12}, \lambda_{6}, \lambda_{7}$ are zero. The $Z_{2}$ symmetry is only softly broken if $m_{12}$ remains non-zero. The $Z_{2}$ charge assignments in the fermion sector leads to four different variants of 2HDM. Consequently, when $\phi_{1}$ coupled only to down-type fermions and $\phi_{2}$ only to up-type fermions, is named as the type II 2HDMs. This discrete symmetry is useful to avoid the large flavor changing neutral currents and appears, as an approximate symmetry, in supersymmetric models. 
We assume all the parameters in the potential to be real. The physical basis is specified by seven parameters which are obtained after the electroweak symmetry breaking. These are the four physical scalar masses $\left(m_{h}, m_{H}, m_{A}\right.$ and $\left.m_{+}\right)$, the total vacuum expectation value (vev) $v=$ $\sqrt{v_{1}^{2}+v_{2}^{2}}, \tan \beta=v_{2} / v_{1}$, and the alignment angle $\cos (\beta-\alpha)$ (here $\alpha$ is the mixing angle in the CP-even sector).

Practically, the complete spectrum of the 2HDMs can be determined from the knowledge of the scalar quartic coupling. For example, if some symmetry principle fixes the qaurtic couplings of Eq. (2.1), e.g. supersymmetry, at some scale $\Lambda_{S}$, then the remaining three bilinear parameters can be solved from the knowledge of $v(=246 \mathrm{GeV}), m_{h}(\simeq 125 \mathrm{GeV})$ and $\tan \beta$ (or alternatively $\cos (\beta-\alpha)$ ). Defining the combination of $\lambda_{i}$ and $\tan \beta$, as follows (see [23, 31] for details):

$$
\begin{aligned}
g_{11}= & \lambda_{1} \cos ^{4} \beta+\lambda_{2} \sin ^{4} \beta+2\left(\lambda_{3}+\lambda_{4}+\lambda_{5}\right) \sin ^{2} \beta \cos ^{2} \beta+4 \lambda_{6} \cos ^{3} \beta \sin \beta+4 \lambda_{7} \sin ^{3} \beta \cos \beta, \\
g_{12}= & \cos \beta \sin \beta\left(\lambda_{2} \sin ^{2} \beta-\lambda_{1} \cos ^{2} \beta+\left(\lambda_{3}+\lambda_{4}+\lambda_{5}\right) \cos 2 \beta\right) \\
& +3\left(\lambda_{7}-\lambda_{6}\right) \sin ^{2} \beta \cos ^{2} \beta+\lambda_{6} \cos ^{4} \beta-\lambda_{7} \sin ^{4} \beta \\
g_{22}= & \left(\lambda_{1}+\lambda_{2}\right) \cos ^{2} \beta \sin ^{2} \beta-2\left(\lambda_{3}+\lambda_{4}\right) \cos ^{2} \beta \sin ^{2} \beta \\
& +\lambda_{5}\left(\sin ^{4} \beta+\cos ^{4} \beta\right)+\left(\lambda_{7}-\lambda_{6}\right) \sin 2 \beta \cos 2 \beta \\
g_{+}= & \frac{1}{2}\left(\lambda_{5}-\lambda_{4}\right) .
\end{aligned}
$$

the diagonalization of the mass matrices determines the couplings in terms of the known $m_{h}$ and $v$.

$$
\begin{aligned}
& g_{11} v^{2}=m_{H}^{2} \cos ^{2}(\beta-\alpha)+m_{h}^{2} \sin ^{2}(\beta-\alpha), \\
& g_{22} v^{2}=m_{H}^{2} \sin ^{2}(\beta-\alpha)+m_{h}^{2} \cos ^{2}(\beta-\alpha)-m_{A}^{2}, \\
& g_{12} v^{2}=\left(m_{h}^{2}-m_{H}^{2}\right) \cos (\beta-\alpha) \sin (\beta-\alpha), \\
& g_{+} v^{2}=m_{+}^{2}-m_{A}^{2},
\end{aligned}
$$

Therefore, once all the quartic couplings $\lambda_{i}$ s are known, the scalar masses and mixing angles can be easily determined from the above relations.

\subsection{Supersymmetric Boundary Conditions}

The MSSM Higgs sector relies upon a two higgs doublet structure. Assuming that all supersymmetric particles are much heavier than the EW scale, the Higgs quartic couplings come from the supersymmetric $D$-terms and, at tree level, are simple functions of the $\mathrm{SU}(2)_{\mathrm{W}}$ and $\mathrm{U}(1)_{\mathrm{Y}}$ gauge couplings $g$ and $g_{Y}$. The matching conditions thus turns out to be [12,32]

$$
\lambda_{1}=\lambda_{2}=\frac{1}{4}\left(g^{2}+g_{Y}^{2}\right), \lambda_{3}=\frac{1}{4}\left(g^{2}-g_{Y}^{2}\right), \lambda_{4}=-\frac{g^{2}}{2}, \lambda_{5}=\lambda_{6}=\lambda_{7}=0,
$$

All the mass terms are also generated at tree level. It is to be noted that the $m_{12}$ term, which breaks the discrete $Z_{2}$ symmetry softly, is related to the bilinear $B \mu$ term in the SUSY potential. Therefore, at tree level, the MSSM leads to a type II 2HDM. The relations of Eq. (2.4) should be understood to hold at a scale $\Lambda_{S}$, where the general 2HDM is matched to the MSSM. Below $\Lambda_{S}$, 
the RG evolution of the 2HDM parameters should be used to obtain the potential at the EW scale. Since the boundary condition $\lambda_{5}=\lambda_{6}=\lambda_{7}=0$ increases the symmetry of the quartic part of the Lagrangian, these couplings will not be generated by the RG evolution and will still be zero at lower energies.

It is worth mentioning that in the MSSM, the $Z_{2}$ symmetry is broken by the $\mu$-term in the superpotential ( $\mu$ being the Higgsino mass parameter), and this breaking affects the higher order matching of all the $\lambda_{i}$ at the scale $\Lambda_{S}$. Specifically, $\lambda_{5,6,7}\left(\Lambda_{S}\right)$ may arise at higher loops but will always be proportional, at least, to $\mu / \Lambda_{S}$ [33], which we can be safely considered as small. Further, since RG evolution cannot generate them, it is reasonable to assume $\lambda_{5} \simeq \lambda_{6} \simeq \lambda_{7} \simeq 0$.

The above assumptions ${ }^{1}$ lead us to only four quartic couplings, that can be determined from the scalar masses and mixings by inverting Eqs. (2.2a)-(2.2d) and using Eq. (2.3) as follows,

$$
\begin{aligned}
& \lambda_{1}=g_{11}+g_{22} \tan ^{2} \beta-2 g_{12} \tan \beta, \\
& \lambda_{2}=g_{11}+g_{22} \cot ^{2} \beta+2 g_{12} \cot \beta, \\
& \lambda_{3}=g_{11}-g_{22}+2 g_{12} \cot (2 \beta)+2 g_{+}, \\
& \lambda_{4}=-2 g_{+} .
\end{aligned}
$$

Once these couplings are determined at the EW scale, including appropriate radiative corrections [22, 34], we can use the 2HDM RGE to check whether their values correspond to the MSSM boundary conditions at a high scale.

\section{Renormalization Group Running and SUSY scale}

To obtain a qualitative understanding of the RG evolution, we can begin by simply using the one loop RGE, checking the stability of these results under higher order corrections a posteriori. At one loop, the RG evolution of the gauge couplings is very simple and can be easily integrated. We will be interested here in the combination $\left(g^{2}+g_{Y}^{2}\right) / 4$ which, in a supersymmetric framework, would fix the boundary values for $\lambda_{1}$ and $\lambda_{2}$. The RG evolution of this combination at one loop is given by, $\mathscr{D}\left(g^{2}+g_{Y}^{2}\right)=\frac{-3 g^{4}+7 g_{Y}^{4}}{8 \pi^{2}}$, where $\mathscr{D} \equiv d / d(\log M)$. Using the EW values $\left(-3 g^{4}+7 g_{Y}^{4}\right) /\left.\left(8 \pi^{2}\right)\right|_{M_{z}} \simeq 0.003$, i.e. this combination remains essentially constant at one loop. On the other hand, the one loop RGE for the quartic couplings depend on the gauge as well as Yukawa couplings [9].

In Fig. 1, we show the two-loop RG running of the quartic couplings $\lambda_{1}, \lambda_{2}$ and the coupling combination $-\left(\lambda_{3}+\lambda_{4}\right)$ for which the boundary values at $\Lambda_{S}$ is fixed at $\left(g^{2}+g_{Y}^{2}\right) / 4$. The dashed line in the figure shows this fixed value. As expected from the one loop RGE of the quartic couplings, only $\lambda_{2}$ deviates siginificanlty from its initial boundary value at the EW scale unlike the other two. The significant contribution from top Yukawa coupling to $\lambda_{2}$ at the low $\tan \beta \sim 1-3$ helps in large deviation of $\lambda_{2}$ at EW scale.

In Fig. 2, we take the bottom-up approach and show the running of $\lambda_{1}$ starting from boundary values set at the EW scale and then evolving upto high scale. This helps to understand how large can $\lambda_{1}$ be at the EW scale to match with the boundary value $\left(g^{2}+g_{Y}^{2}\right) / 4$ at the high scale. In the

\footnotetext{
${ }^{1}$ The correction from the full SUSY spectrum will always be inversely proportional to the high SUSY scale $\Lambda_{S}$ and thus can be safely ignored.
} 

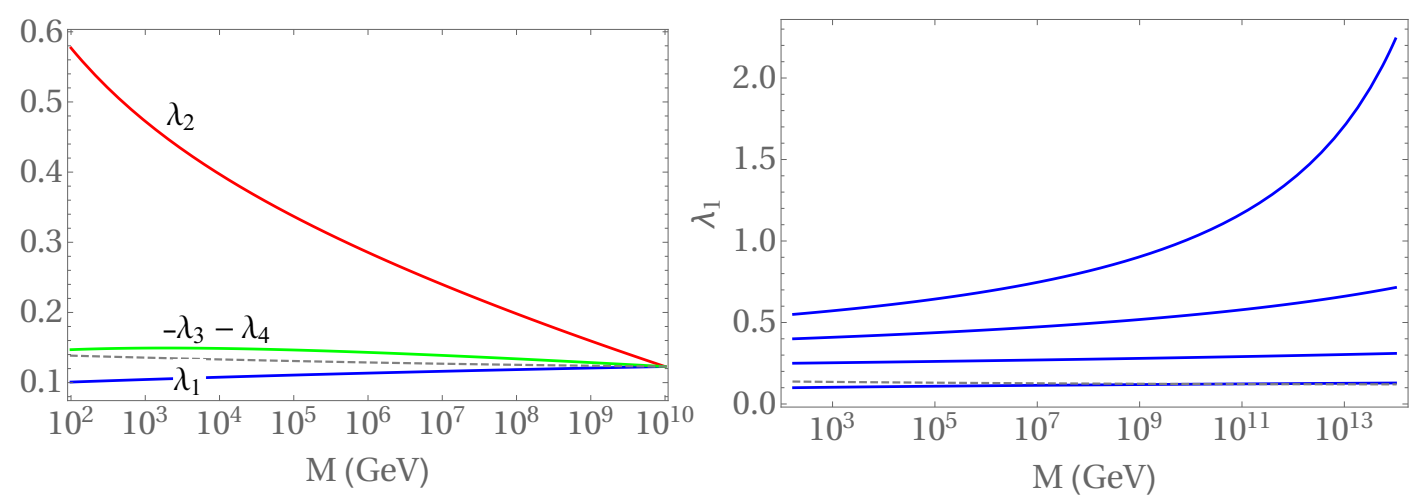

Figure 1: Two-loop $R G$ evolution of $\lambda_{1}, \lambda_{2}$ \&Figure 2: Evolution of $\lambda_{1}$ for different initial val$-\left(\lambda_{3}+\lambda_{4}\right)$ starting from boundary values at $\Lambda_{S}=$ ues (from bottom to top, $\lambda_{1}=0.10,0.25,0.40,0.55$ ), $10^{10} \mathrm{GeV}$ with $\tan \beta=1.7$, as compared to the evo-as compared with $\left(g^{2}+g_{Y}^{2}\right) / 4$ (dashed line), for lution of $\left(g^{2}+g_{Y}^{2}\right) / 4$ (dashed line) [17]. $\quad \tan \beta=1.7$ [17].

figure, initial EW values of the other quartics are set at $\lambda_{2}=0.56, \lambda_{3}=0.015$ and $\lambda_{4}=-0.16$. One should note that the evolution of $\lambda_{1}$ is independent of $\lambda_{2}$ at one loop. We can see that, indeed, $\lambda_{1}$ evolves very little for small values of $\lambda_{1}$ at the EW scale until $\lambda_{1} \leq 0.40$. This conclusion is, in practice, independent of $\tan \beta$ for $\tan \beta \leq 10$. However, since $\lambda_{1}$ grows with the scale, we should expect its value to be slightly smaller than $\left(g^{2}+g_{Y}^{2}\right) / 4 \simeq 0.15$ at the EW scale, if it is to be determined by gauge couplings at the high scale.

Hence, in summary, the following features need to be satisfied in the Higgs quartic measurement at the low scale to have MSSM as the favoured high scale scenario.

- The values of $\lambda_{1}$ and $-\left(\lambda_{3}+\lambda_{4}\right)$, at the EW scale, are in the vicinity of $\left(g^{2}+g_{Y}^{2}\right) / 4 \simeq 0.14$ and must be below $\sim 0.4$.

- The value of $\lambda_{2}$ should then be significantly larger than $\left(g^{2}+g_{Y}^{2}\right) / 4$, due to the large negative contribution to the RGE from the top Yukawa coupling.

- We can get a qualitative estimate of the SUSY scale, $\Lambda_{S}$, as the scale where $\lambda_{2}$ reaches its high scale boundary value, $\left(g^{2}+g_{Y}^{2}\right) / 4$.

\section{SUSY scale determination: Uncertainties and Constraints}

For further illustration of our remarks in the last section, we perform a numerical study of the available parameter space at low energy, provided the quartic couplings have been fixed by the supersymmetric boundary conditions at $\Lambda_{S}$. In Fig. 3, we display the solution regions in terms of physical parameters (mass and mixing angles) for two different choices of $\Lambda_{S}, 10^{10}$ and $10^{16} \mathrm{GeV}$. We only concentrate in the $\tan \beta>1$ region for possible interesting phenomenology following the flavor constraints on type II $2 \mathrm{HDM}$ as flavor data discard $\tan \beta<1$ [35, 36]. The thickness in the allowed parameter region from this analysis, shaded as red, comes from the experimental uncertainties in the Higgs mass $m_{h}=125.0 \pm 0.6 \mathrm{GeV}$ and top pole mass $m_{t}=173 \pm 1 \mathrm{GeV}$. The central continuous line corresponds to their central values. The values of $\tan \beta$ disfavored from 

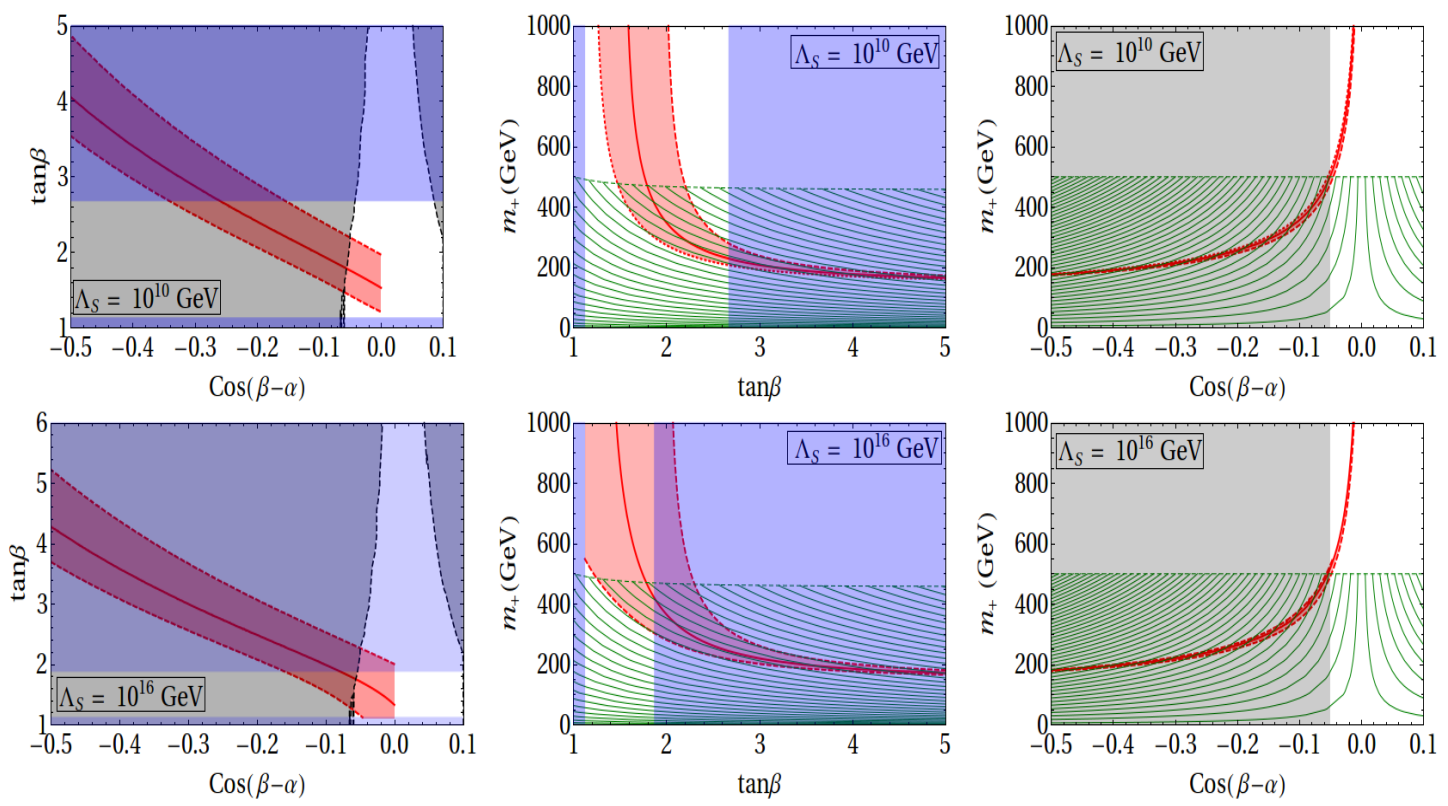

Figure 3: Solution curves in different planes for two different choices of $\Lambda_{S}$ [17]. The widths of the solution regions (in red) arise from $2 \sigma$ experimental uncertainties in $m_{t}$ and $m_{h}$. The regions disallowed from absolute stability (from $M_{Z}$ all the way to $\Lambda_{S}$ ) have been shaded in blue, while the hatched regions are disfavored from $B R(b \rightarrow s \gamma)$ at $95 \%$ C.L. The shaded regions in gray are ruled out from the LHC Higgs data.

absolute stability (from $M_{Z}$ to $\Lambda_{S}$ ) of the scalar potential has been shaded in blue. The hatched region in the middle and right panels of Fig. 3 is disfavored at 95\% C.L. from $\mathrm{BR}(b \rightarrow s \gamma)$ [37]. The gray shaded region in the left panel is forbidden by the Higgs data at 95\% C.L. [38]. The gray region in the right panel, however, represents a disallowed region using a conservative bound on $\cos (\beta-\alpha)$ from the Higgs data[38, 39].

The important features that emerge from our analysis are the following:

- For a large supersymmetric scale, only low $\tan \beta$ values can reproduce the observed Higgs mass. For example, $1.2 \leq \tan \beta \leq 2.2$ for $\Lambda_{S}=10^{10} \mathrm{GeV}$.

- For large $\Lambda_{S}$, an upper limit on $\tan \beta$ is imposed by the requirement of absolute stability, in addition to a lower limit that stability usually offers in a generic 2HDM where the top Yukawa is proportional to $m_{t} /(v \sin \beta)$ [7].

- The $\cos (\beta-\alpha)$ vs $m_{+}$plot shows a strong correlation irrespective of the SUSY scale. This is easily understood as this mixing comes from the diagonalization of the neutral Higgs mass matrix in the Higgs basis, with offdiagonal elements $\mathscr{O}\left(v^{2}\right)$ and a large diagonal entry $\mathscr{O}\left(m_{+}^{2}\right)$.

\subsection{Sensitivity on $\tan \beta$ and $m_{t}$}

As mentioned earlier, $\lambda_{2}$ shows most significant growth than other quartic couplings, if we start from small boundary values at high energy. Hence, $\lambda_{2}$ is our best bet to determine the scale 

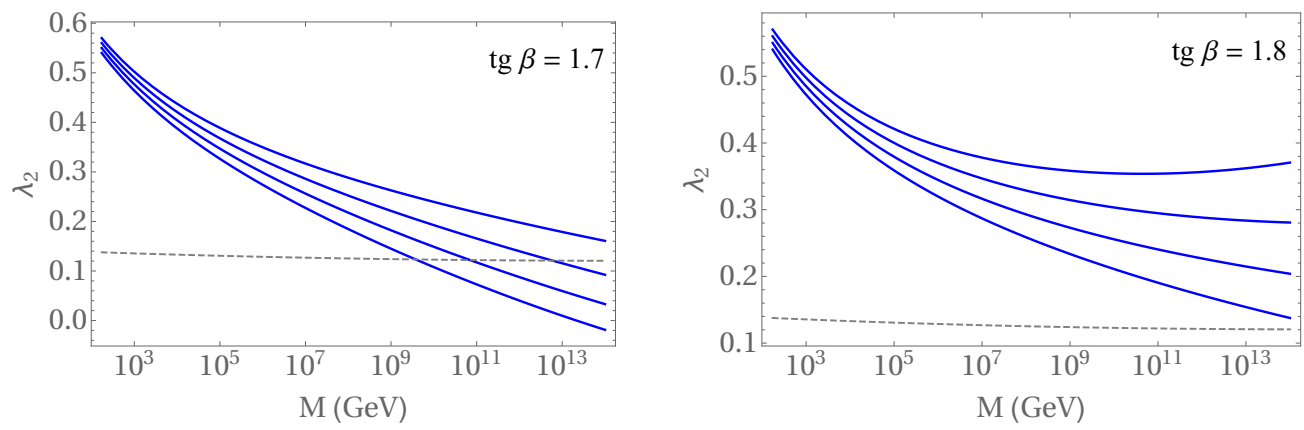

Figure 4: Evolution of $\lambda_{2}$ (low to high scale) as a function of the scale $M$, for different initial values (from bottom to top, $\lambda_{2}=0.54,0.55,0.56,0.57$ ), as compared with $\left(g^{2}+g_{Y}^{2}\right) / 4$ (dashed line) for different $\tan \beta$ values [17].

$\Lambda_{S}$ at which it reaches the boundary value $\left(g^{2}+g_{Y}^{2}\right) / 4$. However, the evolution is highly sensitive to the values of top pole mass $m_{t}$ and $\tan \beta$ at the EW scale, as well as to the initial $\lambda_{2}$ value.

In Fig. 4, we plot the evolution of $\lambda_{2}$ for two close values of $\tan \beta$ and several closely spaced EW values of $\lambda_{2}$ consistent with the observed Higgs mass. It is worth mentioning, that in producing this figure, we choose $m_{t}=173 \mathrm{GeV}$; however, the intrinsic top mass error of about $1 \mathrm{GeV}$ can always be reproduced by a shift in $\tan \beta$. Therefore, the main effect of these uncertainties is a change in the top Yukawa coupling. One can translate both uncertainties as $\Delta \tan \beta=\tan \beta(1+$ $\left.\tan ^{2} \beta\right)\left(\Delta m_{t} / m_{t}\right)$, which shows that $\Delta m_{t}=1 \mathrm{GeV}$ corresponds to $\Delta \tan \beta \sim 0.01$ for $\tan \beta=1$ and $\Delta \tan \beta \sim 0.06$ for $\tan \beta=2$.

It is possible to get an a posteriori explanation for the obtained values of $\lambda_{2}$ and $\tan \beta$ at the EW scale. Under the assumptions of sub-TeV nonstandard scalars and very small $\cos (\beta-\alpha)$, Eq. (2.3) gives $g_{11} v^{2} \simeq m_{h}^{2}$. To a good approximation, we can also write

$$
\lambda_{1}\left(M_{Z}\right) \simeq \lambda_{1}\left(\Lambda_{S}\right)=\lambda_{2}\left(\Lambda_{S}\right)=\frac{\left(g^{2}+g_{Y}^{2}\right)}{4}=-\left\{\lambda_{3}\left(\Lambda_{S}\right)+\lambda_{4}\left(\Lambda_{S}\right)\right\} \simeq-\left\{\lambda_{3}\left(M_{Z}\right)+\lambda_{4}\left(M_{Z}\right)\right\}
$$

Now, using Eq. (2.2a), we obtain

$$
m_{h}^{2}=M_{Z}^{2} \cos ^{2}(2 \beta)+\Delta \lambda_{2} v^{2} \frac{\tan ^{4} \beta}{\left(1+\tan ^{2} \beta\right)^{2}}=M_{Z}^{2}\left(\frac{\tan ^{2} \beta-1}{\tan ^{2} \beta+1}\right)^{2}+\Delta \lambda_{2} v^{2}\left(\frac{\tan ^{2} \beta}{1+\tan ^{2} \beta}\right)^{2},
$$

where, $\Delta \lambda_{2}=\lambda_{2}\left(M_{Z}\right)-\lambda_{2}\left(\Lambda_{S}\right)$. Eq. (4.2) can easily be recognized as the usual expression for the radiatively improved Higgs mass in the MSSM. This implies that the mass of the observed Higgs boson is essentially determined by the RG evolution of $\lambda_{2}$ and the value of $\tan \beta$. For a fixed value of $\tan \beta$, the low energy value of $\lambda_{2}$ is uniquely determined by $m_{h}$. The larger the gap between $\Lambda_{S}$ and $M_{Z}$, the more room $\lambda_{2}$ gets to grow under RG evolution, thereby requiring a smaller $\tan \beta$ to reproduce the observed Higgs mass.

Our analysis shows that an estimate of the SUSY scale is very sensitive to the precise values of the input parameters, especially $\tan \beta$, and as shown in Fig. 4 . We would need to determine $\tan \beta$ at a few percent level to fix $\Lambda_{S}$ precisely. The ambiguity in the determination of the SUSY scale may partly be attributed to a common solution region for $\Lambda_{S}$ in a large range. On the other hand, 
if $\tan \beta$ turns out to be close to 2.2 (say), then one can, for example, make a definitive conclusion that $\Lambda_{S} \leq 10^{10} \mathrm{GeV}$. Such a precise measurement of $\tan \beta$ would, perhaps, require us to wait for the future linear colliders. Nonetheless, the analysis presented in this work is good enough to provide an initial hint for the location of the scale where SUSY is expected to appear.

\section{Conclusions}

We explored an effective $2 \mathrm{HDM}$ arising from a more fundamental theory at a high scale, $\Lambda_{S}$, which fixes the parameters of the Higgs potential. In particular, we have focused on the high-scale MSSM as an example, where the Higgs quartic couplings are determined by the supersymmetry breaking $D$-terms as functions of the gauge couplings. We have found that very high-scale MSSM scenarios are still compatible with the observed Higgs mass for $\tan \beta \sim \mathscr{O}(1)$. We emphasize that our methodology is quite general and can be applied not only to SUSY but to a wide variety of UV scenarios in which all the quartic couplings of the 2HDM potential of Eq. (2.1) are fixed at a high scale, $\Lambda_{S}$. We show that the possibility of determining the supersymmetric scale, $\Lambda_{S}$ can be counted on the RG evolution of $\lambda_{2}$ as the scale where it reaches its boundary value, $\left(g^{2}+g_{Y}^{2}\right) / 4$. However, this strategy crucially depends on whether $\tan \beta$ can be determined with a percent level precision in order to make a reasonable prediction for the MSSM scale; a linear collider would be essential to make further inroads.

\section{Acknowledgements}

This talk is based on work done with Gautam Bhattacharyya, Dipankar Das, Micheal Jay Pérez, Arcadi Santamaria and Oscar Vives. I sincerely thank them for their insightful collaboration. I acknowledge the support from World Premier International Research Center Initiative (WPI), MEXT, Japan. Finally, I thank the Organizers of Corfu2019 for giving me this opportunity.

\section{References}

[1] [CMS], "Combined measurements of the Higgs boson's couplings at $\sqrt{s}=13 \mathrm{TeV}$," CMS-PAS-HIG-17-031.

[2] [ATLAS], "Combined measurements of Higgs boson production and decay using up to $80 \mathrm{fb}^{-1}$ of proton-proton collision data at $\sqrt{s}=13 \mathrm{TeV}$ collected with the ATLAS experiment," ATLAS-CONF-2018-031.

[3] J. F. Gunion and H. E. Haber, Phys. Rev. D 67, 075019 (2003) [hep-ph/0207010].

[4] M. Carena, I. Low, N. R. Shah and C. E. M. Wagner, JHEP 1404, 015 (2014) [arXiv:1310.2248 [hep-ph]].

[5] P. S. Bhupal Dev and A. Pilaftsis, JHEP 1412, 024 (2014) Erratum: [JHEP 1511, 147 (2015)] [arXiv:1408.3405 [hep-ph]].

[6] G. Bhattacharyya and D. Das, Phys. Rev. D 91, 015005 (2015) [arXiv:1408.6133 [hep-ph]].

[7] D. Das and I. Saha, Phys. Rev. D 91, no. 9, 095024 (2015) [arXiv:1503.02135 [hep-ph]].

[8] D. Das and I. Saha, Phys. Rev. D 100, no. 3, 035021 (2019) [arXiv:1904.03970 [hep-ph]]. 
[9] G. C. Branco, P. M. Ferreira, L. Lavoura, M. N. Rebelo, M. Sher and J. P. Silva, Phys. Rept. 516, 1 (2012) [arXiv:1106.0034 [hep-ph]].

[10] G. Bhattacharyya and D. Das, Pramana 87 (2016) no.3, 40 [arXiv:1507.06424 [hep-ph]].

[11] H. P. Nilles, Phys. Rept. 110, 1 (1984).

[12] H. E. Haber and G. L. Kane, Phys. Rept. 117, 75 (1985). doi:10.1016/0370-1573(85)90051-1

[13] J. D. Lykken, hep-th/9612114.

[14] S. P. Martin, Adv. Ser. Direct. High Energy Phys. 21, 1 (2010) [Adv. Ser. Direct. High Energy Phys. 18, 1 (1998)] [hep-ph/9709356].

[15] M. Drees, R. Godbole and P. Roy, "Theory and phenomenology of sparticles: An account of four-dimensional $\mathrm{N}=1$ supersymmetry in high energy physics," Hackensack, USA: World Scientific (2004) $555 \mathrm{p}$

[16] H. Baer and X. Tata, "Weak scale supersymmetry: From superfields to scattering events,"

[17] G. Bhattacharyya, D. Das, M. J. Pérez, I. Saha, A. Santamaria and O. Vives, Phys. Rev. D 97 (2018) no.9, 095018 [arXiv:1712.00791 [hep-ph]].

[18] M. Carena, J. Ellis, J. S. Lee, A. Pilaftsis and C. E. M. Wagner, JHEP 1602, 123 (2016) [arXiv:1512.00437 [hep-ph]].

[19] P. Athron, J. h. Park, T. Steudtner, D. Stöckinger and A. Voigt, JHEP 1701, 079 (2017) [arXiv:1609.00371 [hep-ph]].

[20] F. Staub and W. Porod, Eur. Phys. J. C 77, no. 5, 338 (2017) [arXiv:1703.03267 [hep-ph]].

[21] H. E. Haber, S. Heinemeyer and T. Stefaniak, Eur. Phys. J. C 77, no. 11, 742 (2017) [arXiv:1708.04416 [hep-ph]].

[22] G. Chalons, A. Djouadi and J. Quevillon, Phys. Lett. B 780, 74 (2018) [arXiv:1709.02332 [hep-ph]].

[23] G. Lee and C. E. M. Wagner, Phys. Rev. D 92, no. 7, 075032 (2015) [arXiv:1508.00576 [hep-ph]].

[24] E. Bagnaschi, F. Brümmer, W. Buchmüller, A. Voigt and G. Weiglein, JHEP 1603, 158 (2016) [arXiv:1512.07761 [hep-ph]].

[25] S. A. R. Ellis and J. D. Wells, Phys. Rev. D 96, no. 5, 055024 (2017) [arXiv:1706.00013 [hep-ph]].

[26] J. D. Wells and Z. Zhang, JHEP 1805, 182 (2018) [arXiv:1711.04774 [hep-ph]].

[27] M. E. Machacek and M. T. Vaughn, Nucl. Phys. B 236, 221 (1984).

[28] M. E. Machacek and M. T. Vaughn, Nucl. Phys. B 222, 83 (1983).

[29] M. E. Machacek and M. T. Vaughn, Nucl. Phys. B 249, 70 (1985).

[30] G. Bhattacharyya, D. Das, P. B. Pal and M. N. Rebelo, JHEP 1310, 081 (2013) [arXiv:1308.4297 [hep-ph]].

[31] J. Bernon, J. F. Gunion, H. E. Haber, Y. Jiang and S. Kraml, Phys. Rev. D 92, no. 7, 075004 (2015) [arXiv:1507.00933 [hep-ph]].

[32] D. J. H. Chung, L. L. Everett, G. L. Kane, S. F. King, J. D. Lykken and L. T. Wang, Phys. Rept. 407, 1 (2005) [hep-ph/0312378].

[33] H. E. Haber and R. Hempfling, Phys. Rev. D 48, 4280 (1993) [hep-ph/9307201]. 
[34] J. Braathen, M. D. Goodsell, M. E. Krauss, T. Opferkuch and F. Staub, Phys. Rev. D 97, no. 1, 015011 (2018) [arXiv:1711.08460 [hep-ph]].

[35] O. Deschamps, S. Descotes-Genon, S. Monteil, V. Niess, S. T’Jampens and V. Tisserand, Phys. Rev. D 82, 073012 (2010) [arXiv:0907.5135 [hep-ph]].

[36] D. Das, Int. J. Mod. Phys. A 30, no. 26, 1550158 (2015) [arXiv:1501.02610 [hep-ph]].

[37] M. Misiak and M. Steinhauser, Eur. Phys. J. C 77, no. 3, 201 (2017) [arXiv:1702.04571 [hep-ph]].

[38] J. Bernon, B. Dumont and S. Kraml, Phys. Rev. D 90, 071301 (2014) [arXiv:1409.1588 [hep-ph]].

[39] D. Chowdhury and O. Eberhardt, JHEP 1805, 161 (2018) [arXiv:1711.02095 [hep-ph]]. 